\title{
Supplemental vitamin B-12 enhances the neural response to sensory stimulation in the barrel cortex of healthy rats but does not affect spontaneous neural activity
}

Article

Accepted Version

Kang, S., Hayashi, Y., Bruyns-Haylett, M., Baker, D. K., Boura, M., Wang, H., Karatzas, K.-A., Serra, I., Bithell, A., Williams, C., Field, D. and Zheng, Y. (2019) Supplemental vitamin B-12 enhances the neural response to sensory stimulation in the barrel cortex of healthy rats but does not affect spontaneous neural activity. Journal of Nutrition, 149 (5). pp. 730-737. ISSN 1541-6100 doi: https://doi.org/10.1093/jn/nxz011 Available at https://centaur.reading.ac.uk/81678/

It is advisable to refer to the publisher's version if you intend to cite from the work. See Guidance on citing.

To link to this article DOI: http://dx.doi.org/10.1093/jn/nxz011

Publisher: American Society for Nutrition

All outputs in CentAUR are protected by Intellectual Property Rights law, including copyright law. Copyright and IPR is retained by the creators or other copyright holders. Terms and conditions for use of this material are defined in the End User Agreement. 


\section{www.reading.ac.uk/centaur}

\section{CentAUR}

Central Archive at the University of Reading

Reading's research outputs online 


\section{Supplemental vitamin B-12 enhances the neural}

\section{response to sensory stimulation in the barrel cortex of healthy rats but does not affect spontaneous neural activity}

Sungmin Kang ${ }^{1,7}$, Yurie Hayashi ${ }^{1}$, Michael Bruyns-Haylett ${ }^{2}$, Daniel H. Baker ${ }^{3}$, Marcia Boura ${ }^{4}$, Xuedan Wang ${ }^{4}$, Kimon-Andreas Karatzas ${ }^{4,7}$, Ines Serra ${ }^{5}$, Angela Bithell ${ }^{5}$, Claire Williams ${ }^{6}$, David T. Field ${ }^{7}$, Ying Zheng ${ }^{1,7 *}$

*Corresponding author: Ying Zheng. Tel: +441183787635. Fax: +441189751994. Email:

ying.zheng@reading.ac.uk. Postal address: Biomedical Engineering, School of Biological Sciences, Whiteknights, University of Reading, Reading RG6 7AY, UK

List of all authors' last names: Kang, Hayashi, Bruyns-Haylett, Baker, Boura, Wang, Karatzas, Serra, Bithell, Williams, Field, Zheng.

Word count: 4120

\section{Number of figures: 5}

Number of tables: 0

\section{Supplementary data submitted: 0}

Running title: Vitamin B-12 supplementation enhances the neural signal

${ }^{1}$ Biomedical Engineering, School of Biological Sciences, Whiteknights, University of Reading, Reading RG6 7AY, UK. ${ }^{2}$ Department of Bioengineering, Imperial College, South Kensington Campus, London SW7 2AZ, UK. ${ }^{3}$ Department of Psychology and York Biomedical Research Institute, University of York, Heslington, York, YO10 5DD, UK. ${ }^{4}$ Food and Nutritional Sciences, School of Chemistry, Food and Pharmacy, Whiteknights, University of Reading, Reading RG6 6AP, UK. ${ }^{5}$ Pharmacy, School of Chemistry, Food and Pharmacy, Whiteknights, University of Reading, Reading RG6 7AP, UK. ${ }^{6}$ Psychology, School of Psychology and Clinical Language Sciences, Whiteknights, University of Reading, Reading RG6 6AL, UK. ${ }^{7}$ Centre for Integrative Neuroscience and Neurodynamics (CINN), University of Reading, Reading RG6 6AL, UK.

Abbreviations: B-12, Vitamin B-12; CON: control; CSD, current source density; EEG, electroencephalography; GABA, $\gamma$-aminobutyric acid; LFP, local field potential; MUA, multi-unit activity; PPR, paired-pulse ratio; PSD, power spectral density; SE, standard error.

This work was partly funded by the BBSRC (Grant BB/K010123) and the University of Reading.

Author disclosers: All authors declare no conflicts of interest. 


\section{Abstract}

2 Background: Although vitamin B-12 (B-12) is known to contribute to the structural and

3 functional development of the brain, it is unclear if B-12 supplementation has any beneficial

4 effect in healthy populations in terms of enhanced neurological status of the brain or

5 improved cognitive function.

6 Objectives: We investigated the effect of dietary supplementation of B-12 on the cortical

7 neural activity of well-nourished young adult rats and tested the hypothesis that B-12

8 supplementation in healthy rats may reduce sensory evoked neural activity due to enhanced 9 inhibition.

Methods: Female Lister Hooded rats weighing between $190 \mathrm{~g}$ to $265 \mathrm{~g}$ (2 to 4 months old) were included in the study. The experimental group was fed with B-12 (Cyanocobalamin) enriched water at a concentration of $1 \mathrm{mg} / \mathrm{L}$, and the control (CON) group with tap water for 3 weeks. Animals were then anaesthetised and cortical neural responses to whisker stimulation were recorded in vivo using a multi-channel micro-electrode, from which local field potentials (LFPs) were extracted.

Results: Somatosensory evoked LFP was enhanced $25 \%$ in the B-12 group $(4.13 \pm 0.24 \mathrm{mV})$ compared with the CON group $(3.30 \pm 0.21 \mathrm{mV})(P=0.02)$. Spontaneous neural activity did not differ between groups; frequency spectra at each frequency bin of interest did not pass the cluster-forming threshold at the $5 \%$ significance level.

Conclusions: These findings do not provide evidence supporting the hypothesis of decreased neural activity due to B-12 supplementation. As the spontaneous neural activity was unaffected, the increase in somatosensory evoked LFP may be due to enhanced afferent signal reaching the barrel cortex from the whisker pad, indicating that B-12 supplemented rats may have enhanced sensitivity to sensory stimulation compared to the CON group. We suggest that this enhancement might be the result of lowered sensory threshold, although the underlying mechanism has yet to be elucidated. 
27 Key words

Local field potential, rat barrel cortex, vitamin B-12, dietary supplementation, sensory threshold, GABA.

\section{Introduction}

Vitamin B-12 (B-12) is an essential nutrient, vital for the maintenance of blood and nervous system function. It is a cofactor in the biosynthesis of methionine, a precursor for Sadenosyl-methionine in the brain. S-adenosyl-methionine is a major methyl donor for numerous central nervous system methylation reactions involving neurotransmitters, and plays a crucial role in myelin methylation [1-4].

Given the critical biochemical role that B-12 plays in human metabolic processes and in the synthesis of neurotransmitters, a recent study [5] investigated whether dietary supplementation with a yeast extract rich in B-12 could alter neural activity produced by visual patterns in the brains of healthy subjects. Using electroencephalography (EEG), the researchers observed a reduction in the steady state visual evoked potential for the intervention group compared to the placebo group, and it was speculated that B-12 supplementation in healthy subjects might lead to increased concentration of the inhibitory neurotransmitter $y$-aminobutyric acid (GABA), which in turn could modulate cortical excitation and inhibition. We will refer to this as the 'GABA hypothesis'.

The primary objective of the current study was to investigate whether dietary supplementation with B-12 has a significant effect on cortical neural activity. A set of in vivo electrophysiological experiments were conducted to examine the effect of B-12 supplementation on cortical neural activity of healthy rats (2-4 months old) without B-12 deficiency. If B-12 supplementation could lead to increased global GABA concentration in the brain, the likely effect would be a reduction in both the task-evoked and the spontaneous neural activity [6]. Thus somatosensory evoked local field potential (LFP) as well as 
spontaneous neural activity in rats with and without B-12 intervention were collected and analysed. By examining the temporal dynamics of the evoked LFP, it was possible to assess how B-12 supplementation may have modulated components of synaptic excitation and inhibition in the LFP profile $[7,8]$. To further investigate possible mechanisms underlying the observed changes in evoked LFP responses, we used paired-pulse stimulation to compare the sensory adaptation characteristics of the LFP between the diet groups, as sensory adaptation has been shown to be related to the intensity of stimulation [9].

\section{Materials and Methods}

All experiments were carried out in accordance with United Kingdom Home Office regulations (Animals (Scientific Procedures) Act, 1986) and approved by the Research Ethics Committee at the University of Reading, UK.

\subsection{Animals and diets}

A total of 29 female Lister Hooded rats weighing between $190 \mathrm{~g}$ to $265 \mathrm{~g}$ ( 2 to 4 months old) were included in the study. The strain, gender and age of the rats were chosen based on our previous work $[8,10]$. This choice allowed us to re-use some of our previous data when making comparisons between $\mathrm{CON}$ and $\mathrm{B}-12$ rats. Rats were housed in a temperaturecontrolled room with a 12-h light:dark cycle with ad libitum access to food and water, and were allowed to acclimatise to the animal room conditions and husbandry procedures for 3 days prior to the start of the feeding programme which lasted for 3 weeks.

All rats were fed with standard commercial food (Rat and Mouse No.3 Breeding, RM3(E), 801066, Special Diets Services, UK. The proximate composition: Moisture: $10.00 \%$, Crude Oil: 4.25\%. Crude Protein: 22.39\%. Crude Fibre: 4.21\%. Ash: 7.56\%. Nitrogen Free Extract: $51.20 \%$ ), which has a B-12 (Cyanocobalamin) concentration of $26.78 \mu \mathrm{g} / \mathrm{kg}$, including $17.75 \mu \mathrm{g} / \mathrm{kg}$ supplemented B-12 from manufactured sources. Although this is less than the recommended dietary allowance (RDA) at $50 \mu \mathrm{g} / \mathrm{kg}$ diet for rats [11], it is close to the $\mathrm{B}-12$ concentration in standard feeds used in other studies, and is well above the B-12 
concentration in feeds deficient in $B-12[12,13]$. This was confirmed by the analysis of $B-12$ concentration in serum samples (see Results).

The CON group ( $n=14)$ was fed with fresh water, while the B-12 group $(n=15)$ was fed with B-12 (Cyanocobalamin, Sigma-Aldrich, UK) enriched water. B-12 was added to water incrementally for the first 3 days of the feeding programme at $25 \%, 50 \%$ and $75 \%$ of the final concentration, which was 40 times the RDA for rats [11]. Assuming the daily intake of food and water for adult rats to be approximately $5 \mathrm{~g} / 100 \mathrm{~g}$ and $10 \mathrm{~mL} / 100 \mathrm{~g}$ body weight respectively $[14,15]$, we estimated the RDA of $B-12$ to be $0.25 \mu \mathrm{g} / 100 \mathrm{~g}$ of rat's body weight. To provide $100 \%$ RDA of B-12 through water, the B-12 concentration would be $0.25 \mu \mathrm{g} / 10 \mathrm{~mL}$ water. Thus the final concentration for the intervention was set at $40 \times 0.25 \mu \mathrm{g} / 10 \mathrm{~mL}=$ $10 \mu \mathrm{g} / 10 \mathrm{~mL}$ water, or $1 \mathrm{mg} / \mathrm{L}$ water. We chose $40 \times \mathrm{RDA}$ to be the final concentration in order to ensure the effectiveness of B-12 supplementation for this study. Dosages much higher than this have been used in both rodents and humans without evidence of adverse health effects $[16,17]$. Note that fresh water (without B-12) was not supplied to the B-12 group.

\subsection{Surgery, neural recording and sample collection}

For detailed experimental procedures, the reader is directed to our previous publications [8, $18,19]$ for reference. They are briefly reviewed below.

Following 3 weeks of supplementation, animals were weighed, anaesthetised and operated on following our laboratory's standard surgical procedures. Stimulation was in terms of brief electric current pulses which were applied to the right whisker pad, and the neural activity of the contralateral barrel cortex was recorded via a 16-channel multi-laminar micro-electrode inserted perpendicular to the cortical surface of the barrel cortex. The neural signals thus recorded were typically low-pass filtered below $\sim 500 \mathrm{~Hz}[20]$ to produce the LFP which reflected changes in extra-cellular potentials with respect to a reference potential, and was primarily the weighted sum of post-synaptic activities of the local pyramidal neural 
population. During whisker stimulation, LFP became more negative as positive currents flowed from extracellular space into intracellular space to depolarise principal neurons. The amplitude of the LFP deflection is approximately proportional to the strength of the stimulation [21], and the LFP deflection during the initial timeframe (1 2ms from the onset of the deflection) represents solely the excitatory post-synaptic activity of the local pyramidal neural population $[7,8]$.

A minimum of 100 trials were collected per animal with an inter-trial-interval of at least $5 \mathrm{~s}$. All neural data were sampled at $24.41 \mathrm{kHz}$. Stimulus intensity of $1.2 \mathrm{~mA}$ was used for all animals. After initial analysis which revealed a larger evoked LFP amplitude in the B-12 supplemented group compared with the CON group (see Results), an additional experimental condition with a stimulus intensity of $1.6 \mathrm{~mA}$ was added to four rats in the CON group to investigate if the evoked LFP response under stronger stimulus intensity without B12 supplementation could result in similar amplitude increases. The $1.6 \mathrm{~mA}$ intensity is the strongest intensity previously tested without causing changes in either blood pressure or heart rate of rats under the adopted experimental paradigm [21-23]. The LFP data from these rats were then combined with an existing data set $(n=4)$ collected from previous experiments conducted in our laboratory using identical experimental protocols and stimulus intensities [8]. Thus the total number of rats (in the CON group) subjected to both $1.2 \mathrm{~mA}$ and 1.6mA stimulus intensities was eight.

Finally for a subset of subjects ( $n=9$ /group), additional paired-pulse stimulation at $1.2 \mathrm{~mA}$ was used to investigate if sensory adaptation characteristics could be altered by B-12 supplementation. Stimulus parameters were kept the same for each pulse, while the interpulse-interval was set as $200 \mathrm{~ms}$, with the inter-trial-interval set as 10 s.

At the end of each experiment, the rat was terminated by cervical dislocation. Blood collected via cardiac puncture was centrifuged at $3030 \times \mathrm{g}$ for 6 minutes at room temperature, and serum was then collected and stored at $-80^{\circ} \mathrm{C}$ for further analysis. Brains were extracted, weighed and stored. 
131 Note that only 26 ( $n=13$ group) out of the 29 rats in the study provided usable neural data

132 due to premature death or damage during surgery. Also serum was successfully collected 133 from $21(\mathrm{CON}, \mathrm{n}=10 ; \mathrm{B} 12, \mathrm{n}=11)$ rats only. Serum samples were analysed for cobalamin (B-

134 12) concentration using the Immulite/Immulite 1000 Systems VB Vitamin B12 (Siemens 135 Healthcare Diagnostics Products Ltd) at the Pathology and Diagnostic Laboratories of the 136 Royal Veterinary College based in Hertfordshire, UK. The device uses a solid-phase, competitive chemiluminescent enzyme immunoassay which has an intraassay imprecision (mean \pm standard deviation) of $1308 \pm 77(\mathrm{pg} / \mathrm{mL})$. The standard protocol for the quantitative measurement of B-12 in serum, as detailed in the manufacture's user guide, was followed.

\subsection{Data pre-processing and parameter Estimation}

Neural recordings from the micro-electrode were first pre-processed using our laboratory's standard procedure [8]. Briefly, stimulus artifact was removed, data were zero-meaned at baseline and low pass filtered. Inverse Current Source Density (spline iCSD [24]) analysis was performed to locate the layer IV sink [25] for each data set, and the CSD data were then used to align both the CSD and the LFP data according to their sink locations across animals, with the common sink placed $600 \mu \mathrm{m}$ below the pial surface. We used the realigned LFP time series at channel 7, where the sink was located, to represent the evoked neural activity to whisker pad stimulation, as this channel was located in the cortical layer which was targeted by thalamocortical afferents, with thalamus acting as a relay to deliver tactile response to whisker stimulation to the barrel cortex [26-28]. The evoked LFP was calculated by averaging over 100 trials for each animal. The first negative deflection observed in the evoked LFP was referred to as N1.

In order to compare evoked LFP across groups, the following parameters were extracted after pre-processing to smooth and align the data: (i) the onset of $\mathrm{N} 1$, which was defined as the time at which $\mathrm{N} 1$ exceeded $-0.1 \mathrm{mV}$, with the stimulus onset time assigned as zero; (ii) the initial slope of $\mathrm{N} 1$, which was defined as the slope from $2 \sim 25 \%$ of the $\mathrm{N} 1$ peak amplitude; (iii) the peak amplitude of N1, and (iv) the latency of the $\mathrm{N} 1$ peak. 
For paired-pulse analysis, the amplitude of $\mathrm{N} 1$ of the second pulse was also extracted and the paired-pulse ratio (PPR) was calculated within each trial from

$$
\mathrm{PPR}=\frac{\mathrm{Amp}(\mathrm{N} 1 \text { of second pulse })}{\mathrm{Amp}(\mathrm{N} 1 \text { of first pulse })}
$$

\subsection{Frequency domain analysis}

To investigate possible mechanisms giving rise to differences in evoked LFP responses across diet groups, we checked the anaesthetic levels during the recording period to ensure that they were not significantly different between groups, as it has been shown that sensory evoked LFP is sensitive to the level of anaesthesia, and that the anaesthetic level is reflected in the resting state PSD within the frequency range $1 \sim 8 \mathrm{~Hz}[29,30]$. Thus the resting state PSD below $8 \mathrm{~Hz}$ was used to compare the anaesthetic depth between groups. To compute the resting state PSD for each trial, we used the resting state LFP data 0.9 4.9s post stimulation, down-sampled the data to $10 \mathrm{KHz}$, and calculated PSD in Matlab ${ }^{\mathrm{TM}}$ via Welch's method (Hamming window, 50\% overlap).

It is also well known that sensory evoked neural activity is closely influenced by spontaneous activity in the same cortical region [31-33]. One possible explanation for differences in evoked LFP responses between diet groups could be changes in spontaneous subthreshold activity and/or spontaneous spiking activity due to B-12 supplementation. Thus we extended the resting state PSD calculation to include frequencies up to $3000 \mathrm{~Hz}$ to cover both the subthreshold $(8 \sim 500 \mathrm{~Hz})$ neural activity and the multi-unit activity (MUA, 500 3000Hz) [20].

\subsection{Statistical analysis}

Throughout the analysis, the significance level $\alpha$ was set at 0.05 . Group analysis was performed to compare various measurements and parameters extracted from field potential recordings between the two diet groups using the two-tailed two-sample Student's t-test under the assumption that the sampling distribution of the mean was normally distributed. Parameters were presented as mean \pm standard error (SE). To compare the N1 amplitude in 
response to two stimulus intensities applied to the same rat, the two-tailed paired-sample Student's t-test was used.

To compare the ratios of brain weight to body weight across the groups, the non-parametric Wilcoxon rank-sum test was used to test for equal medians, as the ratio of two normally distributed variables is no longer normally distributed.

For comparison of PSDs over the frequency range $1 \sim 3000 \mathrm{~Hz}$, a non-parametric cluster correction procedure $[34,35]$ was used to determine significant clusters across the frequency range while controlling for multiple comparisons. This involved conducting an independent two-sample t-test at each frequency bin (width $=1 \mathrm{~Hz}$ ) to compare responses in the $\mathrm{CON}$ and the $\mathrm{B}-12$ groups. Tests that were significant at $P<0.05$ were aggregated into clusters across adjacent frequency bins. The summed t-statistic for each cluster was compared to a null distribution generated by resampling the data from the largest cluster 10,000 times with randomly assigned group labels, and recalculating the summed t-statistic. Clusters which fell outside of the empirical $95 \%$ confidence intervals of the null distribution were considered significant.

Finally, we assumed no significant bias in the weight of the rats between the two groups at the start of the feeding programme. This was reasonable based on the fact that all rats, weighing between $175 \sim 224 \mathrm{~g}$, were purchased from the same source (Charles River, UK) on 8 occasions (4 pseudorandom occasions per diet group) across a 20-month period. It should be noted that rats were only weighed once immediately prior to surgery.

\section{Results}

\subsection{B-12 serum concentration, body and brain weights}

The serum cobalamin concentration was $98 \%$ greater in the B-12 group compared with the CON group $(P<0.01)$ (Figure 1A). The concentration in the CON group was within the normal range for rats $[13,36,37]$, thus confirming that they were not deficient in $\mathrm{B}-12$. There 
was no significant difference between the final body weight (Figure 1B) and brain weight

(Figure 1C), and the brain/body weight ratio (Figure 1D) between the two diet groups. Thus our results suggest that, assuming no significant weight difference across diet groups at the start of the feeding programme, B-12 supplementation did not significantly change body weight, brain weight, or the ratio between them.

\subsection{Amplitude of evoked LFP was increased by B-12 supplementation}

Along the cortical depth, the B-12 group had a larger LFP response (Figure 2A) and a correspondingly stronger sink/source pair (Figure 2B) compared to the CON group. These were reflected in the brighter blue colour associated with the B-12 group images. Time series of the evoked LFP responses in the layer IV sink are displayed in Figure 2C. The amplitude of $\mathrm{N} 1$ for the $\mathrm{B}-12$ group was $25.2 \%$ larger than that of the CON group $(P=0.02)$ (Figure 2D), while the latency of the N1 peak for the B-12 group was significantly shorter than the CON group $(P=0.03)$ (Figure 2E). In addition, the initial slope of $\mathrm{N} 1$ for the $\mathrm{B}-12$ group was significantly steeper than the CON group $(P<0.01)$ (Figure 2F), however the onset of $\mathrm{N} 1$ was not significantly different $(P=0.39)$ (Figure $\mathbf{2 G}$ ). Together these characteristics suggested that the dynamics of the evoked LFP response for the B-12 group were faster, reflected in the steeper initial slope, and stronger, in terms of the N1 amplitude, compared to the CON group. However the onset of N1 was not significantly different between the diet groups, with the important implication that B-12 supplementation for 3 weeks did not significantly change the transmission speed of the afferent neural signal arriving at the barrel cortex from the whisker pad.

\subsection{Sensory adaptation was weakened by B-12 supplementation}

Sensory adaptation characteristics of neural responses were investigated using the pairedpulse stimulus paradigm, results of which are shown in Figures 3A and 3B for CON and B12 groups respectively. The PPR for the B-12 group was $21.9 \%$ higher than the CON group $(P=0.04)$ (Figure 3C), indicating that the second pulse was significantly less adapted for the 
B-12 group than the CON group. Therefore, despite a higher amplitude of the first evoked LFP pulse in the B-12 group compared to that of the CON group, the recovery of the second pulse (200ms apart) was faster in the B-12 group.

\subsection{Resting state power spectral density (PSD) analysis}

238 There was a clear overlap of PSDs between individuals in the CON group and those in the B-12 group, indicating no significant difference in either the depth of anaesthesia (Figure 4A), or the subthreshold and MUA neural activity (Figure 4B) between the two diet groups. The nonparametric cluster correction analysis showed that, across all frequency bins, the maximum absolute t-statistic was 1.84 , less than the critical t-value of 2.06 ( $n=13 /$ group, degree of freedom=24) for significance at the $5 \%$ level, further confirming that there was no significant difference in PSDs between the two diet groups in the frequency range $1 \sim 3000 \mathrm{~Hz}$.

\subsection{Effect of stimulus intensity}

247 For rats without B-12 supplementation, the evoked LFP amplitude to the $1.6 \mathrm{~mA}$ stimulation was $13.9 \%$ higher than that to the $1.2 \mathrm{~mA}$ stimulation $(P<0.01)$ (Figure $5 \mathrm{~A})$. For comparison, we re-plotted the LFP responses of CON and B-12 groups to the 1.2mA stimulation (Figure 5B). As stated previously in Section 3.2, the N1 amplitude for the B-12 group was $25.2 \%$ higher than that of the CON group.

\section{Discussion}

To the best of our knowledge, this is the first study to show that healthy rats supplemented with B-12 demonstrate an increase in sensory evoked synaptic activity in the somatosensory cortex. We discuss here possible mechanisms underlying the observed phenomena and 256 their implications for future research. 
It is well-known that B-12 plays a crucial role in myelin methylation. Recent research further suggests that the myelin sheath is more than an inert insulating membrane structure [38-40]. A study on rat somatic sensorimotor system has shown that the structure of myelin sheath in the spinal cord underwent changes throughout the aging process [41]. Furthermore myelination properties have been shown to be regulated by neuronal activity and the environment $[42,43]$. It is therefore plausible that in young adult rats, as used in our study, myelination properties such as myelin sheath length and/or thickness could be altered within a 3-week period, and that the increased neural response described here could be the result of strengthened myelination of neurons in B-12 rats. However, the onset of the N1 deflection in our data across the diet groups did not differ significantly (Figure 2G), suggesting that the neuron conduction velocity was not changed by the supplementation. However, we also recognise that there is a minimum difference of onset that could be detected in our measurement at a $5 \%$ level of significance. This can be estimated using the two-sample tstatistic:

$$
\frac{\bar{x}_{1}-\bar{x}_{2}}{\sqrt{s_{1}^{2} / n_{1}+s_{2}^{2} / n_{2}}}=t_{n_{1}+n_{2}-2,0.05}
$$

With the standard errors of onset shown in Figure $2 \mathrm{G}(\mathrm{CON}$ : SE $=0.08 \mathrm{~ms}$; $\mathrm{B}-12$ : $\mathrm{SE}=0.06 \mathrm{~ms}$; $n=13 /$ group), we found this difference to be $0.21 \mathrm{~ms}$. In other words, if the onset difference between the two groups was $0.21 \mathrm{~ms}$ or less, we would not be able to detect it at a $5 \%$ level of significance.

\subsection{B-12 supplementation and the 'GABA hypothesis'}

We are not aware of any study providing evidence linking dietary supplementation of B-12 to changes in GABA in the brain. However using intracerebroventricular infusion, Ikeda et al [44] investigated the effect of B-12 on circadian pace-making in rodents and found that B-12 infusion significantly increased the content of GABA in the suprachiasmatic nucleus of the hypothalamus, while the content of the excitatory neurotransmitter glutamate in the same region was significantly decreased. The authors speculated that B-12 may modulate the 
metabolism of GABA and glutamate by facilitating glutamic acid decarboxylase activity. In addition, a recent human study investigated the effect of dietary intervention of a yeast extract substance on steady state visual evoked potentials (VEPs), and found reduced neural responses in the diet group compared to the placebo group [5]. As the yeast substance was richer in B-12 in comparison to the placebo substance, the researchers suggested that the observed reduction could be the result of increased GABA concentration in the brain due to dietary supplementation of B-12.

Direct comparison of our study to the above human study is not possible, not least because the stimulus paradigms used in the two studies were very different. However if dietary supplementation with B-12 increased the global GABA concentration in the brain, the likely effect on the spontaneous as well as task-evoked neural activity would be a reduction in both [6]. PSD analysis of our data during resting state showed no significant difference between the two groups over the frequency range $1 \sim 3000 \mathrm{~Hz}$ (Figure 4). In addition, the evoked LFP for the B-12 group showed significantly increased amplitude and faster temporal dynamics. Both of these observations could be taken as evidence against the GABA hypothesis.

GABA concentration in rat brain can be measured using techniques such as Gas Chromatography-Mass Spectrometry, immunohistochemistry and magnetic resonance spectroscopy. We plan to conduct some of these tests for our future studies.

\subsection{B-12 enhanced LFP may implicate enhanced sensitivity to sensory stimulation}

Figure $2 \mathrm{~F}$ showed that the initial slope of $\mathrm{N} 1$ for the $\mathrm{B}-12$ group is steeper than that of the CON group. Based on our previous study [8], this suggests faster excitatory post-synaptic activity for the B-12 group. On the other hand we didn't observe significant difference in the resting state neural activity between the groups. The scenario is analogous to the barrel cortex responding to whisker stimulation with two levels of intensity, the stimulus with stronger intensity will evoke a higher LFP response amplitude than that evoked by the lower intensity stimulus, while the resting state LFP will be unaffected by stimulus strength $[8,21$, 
23]. In other words, the enhanced LFP response for the B-12 group could be due to enhanced thalamo-cortical afferent signal, suggesting that B-12 supplementation in wellnourished rats may have enhanced the sensitivity of neurons to sensory stimulation in the lemniscal pathway linking peripheral nerves in the whisker pad to neurons in the thalamus. This is further supported by our results on sensory adaptation. The mechanism underlying sensory adaptation and stimulus strength was studied in detail by Ganmor et al [9] who demonstrated that stronger whisker stimulation produced weaker sensory adaptation in the somatosensory cortex of rodent. They pinpointed the source of this weaker adaptation to neurons in the brainstem trigeminal complex and argued that such coding strategy may be used to discriminate stimulus intensities during adaptation in order to counterbalance the effect of short-term synaptic depression in the thalamus and subsequently in the cortex. Based on their work, our observed weaker sensory adaptation in the B-12 group could be due to neurons in the brainstem responding more strongly to the same stimulus compared to the CON group, with faster recovery and subsequently less adaptation to the second stimulus. Further experiments will be needed to confirm this by using a wider range of stimulus intensities with neural recordings from the thalamus and the brainstem of both diet groups.

\subsection{B-12 supplementation and sensory threshold}

Changes in sensory evoked potentials have been linked to changes in sensory threshold. Lund et al [45] found that, post-surgery, sensory threshold to cutaneous electrical stimulation was increased, while the peak-to-peak amplitudes of somatosensory evoked potentials were decreased significantly. A more recent study on pain-threshold and aggressiveness found that individuals who more often behave aggressively had a higher pain threshold, and aggressiveness was negatively correlated to the amplitude of pain-related evoked potentials [46]. In auditory research, evoked potentials have been found to correlate with auditory signal detection, specifically the amplitude of auditory evoked potentials associated with correctly detected signals were found to be much higher than those corresponding to falsely 
reported signals, undetected signals or correctly reported non-signals [47]. Furthermore, sensory thresholds can be lowered by training. Using the human visual system, Skrandies et al [48] demonstrated that sensory threshold decreased during repeated presentation of visual hyper-acuity stimuli. This was accompanied by significantly larger amplitude of VEPs. Interestingly, they also observed significantly shorter peak latency in VEPs post training, which agrees with the shorter N1 peak latency for the B-12 group (Figure 2E).

Based on this body of literature, we suggest that B-12 supplementation may have the effect of lowering the sensory threshold, thus enhancing the sensitivity of neurons to sensory stimulation.

\subsection{Future work}

The conclusion of the study is limited by several shortcomings. One is that the concentration of GABA in the brain was not measured, thus we were unable to confirm if the sensory evoked LFP difference between the diet groups was related to the different levels of GABA concentration. The other is that we did not measure B-12 concentration in the brain, although its concentrations in serum samples were obtained. Furthermore, cognitive correlates of B-12 supplementation were not assessed. We plan to incorporate these measurements in future studies to further elucidate the role that B-12 may play in shaping the neurological and cognitive functions of the brain.

Dietary supplementation of B12 is inexpensive and non-toxic. If it can be demonstrated to slow down age-related cognitive decline through increased responsiveness to sensory stimulation, it will have significant impact on the well-being of older people, and generate considerable economic as well as public health benefits.

\section{Acknowledgement}

We thank Prof. Simon Andrews, Dr. Stephen Elmore, Dr. Peter Harris, Ms. Amanpreet Kaur, Mr. Andrew Cripps and the BRU at the University of Reading, and Ms Sue Rodway at the 
Royal Veterinary College based in Hertfordshire, UK for their assistance during the project.

The authors' contributions to the manuscripts are as follows: D.T.F., M.B-H., Y.Z. and D.H.B. conceived the project. Y.Z., M.B-H. and C.W. designed the research. S.K., Y.H., M.B-H. and Y.Z. collected data. M.B., X.W., K.K., I.S. and A.B. provided essential materials. Y.Z., S.K., M.B-H., D.H.B. analysed data. Y.Z. wrote the manuscript. D.T.F., C.W., A.B., M.B-H., D.H.B. and I.S. contributed to the editing of the manuscript. Y.Z. had primary responsibility for the final content. All authors have read and approved the final manuscript.

\section{References}

1. Young SN, Shalchi M: The effect of methionine and S-adenosylmethionine on Sadenosylmethionine levels in the rat brain. $J$ Psychiatry Neurosci 2005, 30:44-48.

2. Gröber U, Kisters K, Schmidt J: Neuroenhancement with Vitamin B12Underestimated Neurological Significance. Nutrients 2013, 5:5031-5045.

3. Kennedy DO: B Vitamins and the Brain: Mechanisms, Dose and Efficacy-A Review. Nutrients 2016, 8:68.

4. Porter K, Hoey L, Hughes FC, Ward M, McNulty H: Causes, Consequences and Public Health Implications of Low B-Vitamin Status in Ageing. Nutrients 2016, 8.

5. Smith A, K., Wade A, R. , Penkman K, E. H. , Baker D, H. : Dietary modulation of cortical excitation and inhibition. J Psychopharmacol (Oxf) 2017, 31:632-637.

6. Duncan NW, Wiebking C, Northoff G: Associations of regional GABA and glutamate with intrinsic and extrinsic neural activity in humans-A review of multimodal imaging studies. Neurosci Biobehav Rev 2014, 47:36-52.

7. Zheng Y, Luo JJ, Harris S, Kennerley A, Berwick J, Billings SA, Mayhew J: Balanced excitation and inhibition: Model based analysis of local field potentials. Neuroimage 2012, 63:81-94. 
8. Bruyns-Haylett M, Luo J, Kennerley AJ, Harris S, Boorman L, Milne E, Vautrelle N, Hayashi Y, Whalley BJ, Jones M, et al: The neurogenesis of P1 and N1: A concurrent EEG/LFP study. Neuroimage 2017, 146:575-588.

9. Ganmor E, Katz Y, Lampl I: Intensity-Dependent Adaptation of Cortical and Thalamic Neurons Is Controlled by Brainstem Circuits of the Sensory Pathway. Neuron 2010, 66:273-286.

10. Mayhew J, Zheng Y, Hou YQ, Vuksanovic B, Berwick J, Askew S, Coffey P: Spectroscopic analysis of changes in remitted illumination: the response to increased neural activity in brain. Neuroimage 1999, 10:304-326.

11. Council NR: Nutrient Requirements of Laboratory Animals,: Fourth Revised Edition, 1995. Washington, DC: The National Academies Press; 1995.

12. Jaffé WG: Requirements of Rats for Vitamin B12 During Growth, Reproduction and Lactation. The Journal of Nutrition 1956, 59:135-146.

13. Birn $\mathrm{H}, \mathrm{Nex} \varnothing \mathrm{E}$, Christensen El, Nielsen R: Diversity in rat tissue accumulation of vitamin B12 supports a distinct role for the kidney in vitamin B12 homeostasis. Nephrology Dialysis Transplantation 2003, 18:1095-1100.

14. Hubert M-F, Laroque P, Gillet J-P, Keenan KP: The Effects of Diet, ad Libitum Feeding, and Moderate and Severe Dietary Restriction on Body Weight, Survival, Clinical Pathology Parameters, and Cause of Death in Control Sprague-Dawley Rats. Toxicol Sci2000, 58:195-207.

15. Beale KEL, Murphy KG, Harrison EK, Kerton AJ, Ghatei MA, Bloom SR, Smith KL: Accurate Measurement of Body Weight and Food Intake in Environmentally Enriched Male Wistar Rats. Obesity 2011, 19:1715-1721.

16. Richardson LR, Brock R: Studies of Reproduction in Rats Using Large Doses of Vitamin B12 and Highly Purified Soybean Proteins. The Journal of Nutrition 1956, 58:135-145. 
17. Eussen SM, de Groot LM, Clarke R, et al.: Oral cyanocobalamin supplementation in older people with vitamin b12 deficiency: A dose-finding trial. Arch Intern Med 2005, 165:1167-1172.

18. Boorman L, Harris S, Bruyns-Haylett M, Kennerley A, Zheng Y, Martin C, Jones M, Redgrave P, Berwick J: Long-Latency Reductions in Gamma Power Predict Hemodynamic Changes That Underlie the Negative BOLD Signal. The Journal of Neuroscience 2015, 35:4641-4656.

19. Kang S, Bruyns-Haylett M, Hayashi Y, Zheng Y: Concurrent Recording of Colocalized Electroencephalography and Local Field Potential in Rodent. Journal of Visualized Experiments : JoVE 2017:56447.

20. Einevoll GT, Kayser C, Logothetis NK, Panzeri S: Modelling and analysis of local field potentials for studying the function of cortical circuits. Nat Rev Neurosci 2013, 14:770-785.

21. Jones M, Hewson-Stoate N, Martindale J, Redgrave P, Mayhew J: Nonlinear coupling of neural activity and CBF in rodent barrel cortex. Neuroimage 2004, 22:956-965.

22. Mayhew J, Johnston D, Berwick J, Jones M, Coffey P, Zheng Y: Spectroscopic analysis of neural activity in brain: Increased oxygen consumption following activation of barrel cortex. Neuroimage 2000, 12:664-675.

23. Hewson-Stoate N, Jones M, Martindale J, Berwick J, Mayhew J: Further nonlinearities in neurovascular coupling in rodent barrel cortex. Neuroimage 2005, 24:565-574.

24. Pettersen KH, Devor A, Ulbert I, Dale AM, Einevoll GT: Current-source density estimation based on inversion of electrostatic forward solution: Effects of finite extent of neuronal activity and conductivity discontinuities. $J$ Neurosci Methods 2006, 154:116-133. 
25. Mitzdorf U: Current source-density method and application in cat cerebral cortex: investigation of evoked potentials and EEG phenomena. Physiol Rev 1985, 65:37-100.

26. Feldmeyer D: Excitatory neuronal connectivity in the barrel cortex. Frontiers in neuroanatomy 2012, 6:24-24.

27. Constantinople CM, Bruno RM: Deep cortical layers are activated directly by thalamus. Science (New York, NY) 2013, 340:1591-1594.

28. Mease RA, Krieger P, Groh A: Cortical control of adaptation and sensory relay mode in the thalamus. Proceedings of the National Academy of Sciences 2014 111:6798-6803.

29. Friedberg MH, Lee SM, Ebner FF: Modulation of Receptive Field Properties of Thalamic Somatosensory Neurons by the Depth of Anesthesia. J Neurophysiol 1999, 81:2243-2252.

30. Devonshire IM, Grandy TH, Dommett EJ, Greenfield SA: Effects of urethane anaesthesia on sensory processing in the rat barrel cortex revealed by combined optical imaging and electrophysiology. Eur J Neurosci 2010, 32:786797.

31. Petersen $\mathrm{CCH}$, Hahn TTG, Mehta M, Grinvald A, Sakmann B: Interaction of sensory responses with spontaneous depolarization in layer 2/3 barrel cortex. Proc Natl Acad Sci U S A 2003, 100:13638-13643.

32. Schölvinck ML, Friston KJ, Rees G: The influence of spontaneous activity on stimulus processing in primary visual cortex. Neuroimage 2012, 59:2700-2708.

33. He BJ: Spontaneous and Task-Evoked Brain Activity Negatively Interact. The Journal of Neuroscience 2013, 33:4672-4682.

34. Maris E, Oostenveld R: Nonparametric statistical testing of EEG-and MEG-data. J Neurosci Methods 2007, 164:177-190.

35. Baker DH: Decoding eye-of-origin outside of awareness. Neuroimage 2017, 147:89-96. 
36. Sivakumar B, Nath N, Nath MC: Effect of Various High Protein Diets on Vitamin B12 Status in Rats. The Journal Of Vitaminology 1969, 15:151-154.

37. Nix WA, Zirwes R, Bangert V, Kaiser RP, Schilling M, Hostalek U, Obeid R: Vitamin B status in patients with type 2 diabetes mellitus with and without incipient nephropathy. Diabetes Res Clin Pract 2015, 107:157-165.

38. Simons M, Nave K-A: Oligodendrocytes: Myelination and Axonal Support. Cold Spring Harbor perspectives in biology, 8:a020479-a020479.

39. Young KM, Psachoulia K, Tripathi RB, Dunn S-J, Cossell L, Attwell D, Tohyama K, Richardson WD: Oligodendrocyte dynamics in the healthy adult CNS: evidence for myelin remodeling. Neuron $2013,77: 873-885$

40. Fields RD: White matter in learning, cognition and psychiatric disorders. Trends Neurosci 2008, 31:361-370.

41. Xie F, Liang $\mathrm{P}, \mathrm{Fu} \mathrm{H}$, Zhang J, \&, Chen J: Effects of normal aging on myelin sheath ultrastructures in the somatic sensorimotor system of rats. Molecular Medicine Reports 2014, 10:459-466.

42. Michalski J-P, Kothary R: Oligodendrocytes in a Nutshell. Frontiers in cellular neuroscience 2015, 9:340-340.

43. Gibson EM, Purger D, Mount CW, Goldstein AK, Lin GL, Wood LS, Inema I, Miller SE, Bieri G, Zuchero JB, et al: Neuronal activity promotes oligodendrogenesis and adaptive myelination in the mammalian brain. Science (New York, NY) 2014, 344:1252304-1252304.

44. Ikeda M, Azuma S, Inoue S: Vitamin B12 enhances GABA content but reduces glutamate content in the rat suprachiasmatic nucleus. American Journal of Physiology-Regulatory, Integrative and Comparative Physiology 1997, 273:R359R363.

45. Lund $\mathrm{C}$, Hansen $\mathrm{OB}$, Kehlet $\mathrm{H}$ : Effect of surgery on sensory threshold and somatosensory evoked potentials after skin stimulation. Br J Anaesth 1990, 65:173-176. 
46. Ring C, Kavussanu M, Willoughby A: Pain thresholds, pain-induced frontal alpha activity and pain-related evoked potentials are associated with antisocial behavior and aggressiveness in athletes. Psychology of Sport and Exercise 2016, 22:303-311.

47. Hillyard SA, Squires KC, Bauer JW, Lindsay PH: Evoked Potential Correlates of Auditory Signal Detection. Science 1971, 172:1357-1360.

48. Skrandies W, Lang G, Jedynak A: Sensory thresholds and neurophysiological correlates of human perceptual learning. Spat Vis 1996, 9:475-489. 


\section{Figure legends}

FIGURE 1. Serum cobalamin concentration (A), final body $(B)$ and brain weights $(C)$, and the brain/body weight ratio (D) in adult female rats that did not or did consume B-12 for 3 weeks. Values are means \pm SEs. For serum samples, CON, $n=10 ; B-12, n=11$. For body and brain weights, CON, $\mathrm{n}=14 ; \mathrm{B}-12, \mathrm{n}=15$. Asterisks indicate different from $\mathrm{CON}$ : ${ }^{* *} P<0.01$. B-12, vitamin B12; CON, control.

FIGURE 2. Neural responses and associated parameters in adult female rats that did not or did consume B-12 for 3 weeks. (A) Mean LFPs displayed as images for the two diet groups. Cortical depth is along the vertical axis, with the top of the image being $200 \mu \mathrm{m}$ below pia mater. Time is along the horizontal axis, with the black triangle indicating stimulus onset. (B) Similar to (A) but CSD of the two diet groups. (C) Mean evoked LFP time series of the two diet groups. Shadows indicate SE. Stimulus onset is at $t=0$. (D) The amplitude, (E) the latency, $(F)$ the initial slope, and $(G)$ the onset of $N 1$. Values are means $\pm S E s, n=13 /$ group. Asterisks indicate different from $\mathrm{CON}:{ }^{*} P<0.05 ;{ }^{* *} P<0.01$. B-12, vitamin B12; CON, control; CSD, current source density; LFP, local field potential.

FIGURE 3. Mean LFP responses to paired-pulse stimulation in adult female rats that did not (A) and did (B) consume B-12 for 3 weeks. Shadows indicate SE. Stimulus onsets are at $t=0$ and $t=200 m s$ respectively. (C) PPR of the two diet groups. Values are means \pm SEs, $\mathrm{n}=9$ /group. Asterisks indicate different from $\mathrm{CON}$ : ${ }^{*} P<0.05$. B-12, vitamin B12; CON, control; LFP, local field potential; PPR, paired-pulse ratio.

FIGURE 4. Mean resting state PSD in the frequency range $1 \sim 8 \mathrm{~Hz}(A)$, and $8 \sim 3000 \mathrm{~Hz}(B)$ of adult female rats that did not or did consume B-12 for 3 weeks. Individual subject's PSD are also displayed, $n=13 /$ group. B-12, vitamin B12; CON, control; PSD, power spectral density.

FIGURE 5. (A) Mean LFP responses to whisker stimulation at intensities 1.2mA $(n=13)$ and 1.6mA $(n=8)$ respectively for adult female rats that did not consume B-12. (B) Mean LFP 
responses of the two diet groups at the same stimulus intensity of $1.2 \mathrm{~mA}, \mathrm{n}=13 / \mathrm{group}$. Error bars indicate SEs. B-12, vitamin B12; CON, control; LFP, local field potential.

Figure 1
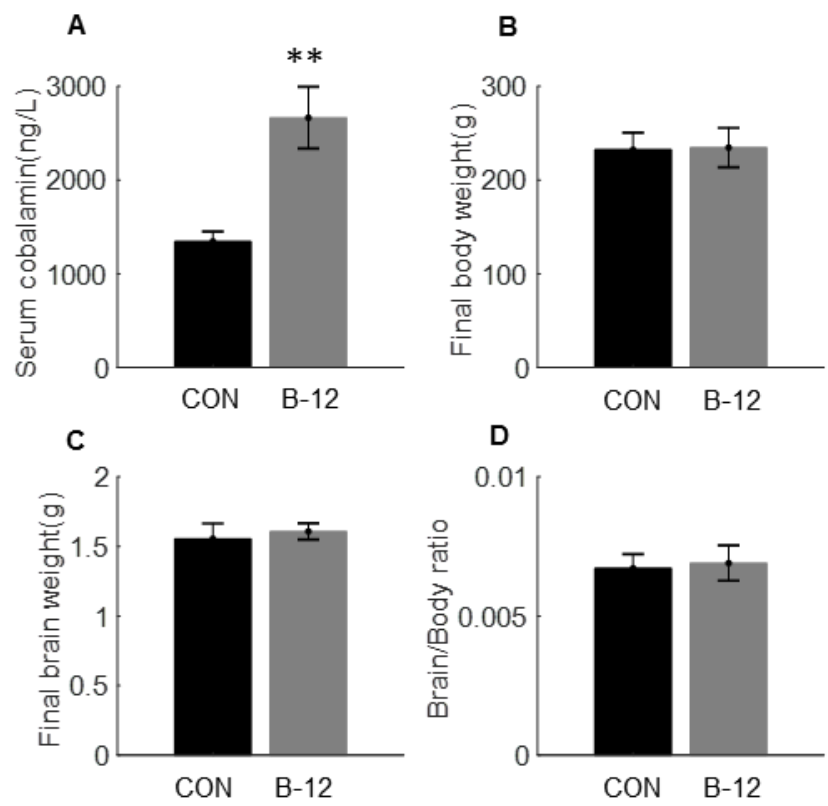
Figure 2
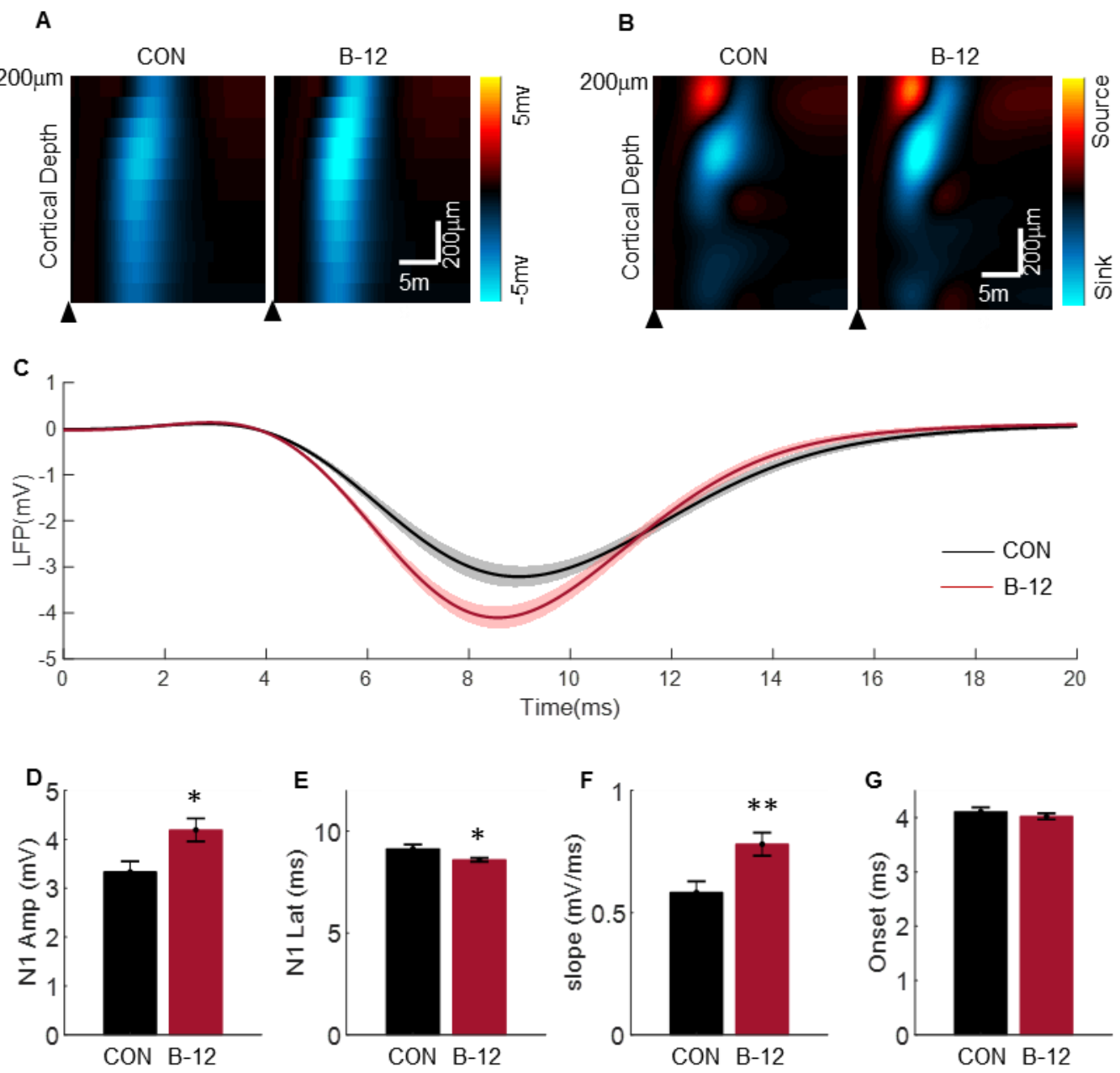
Figure 3
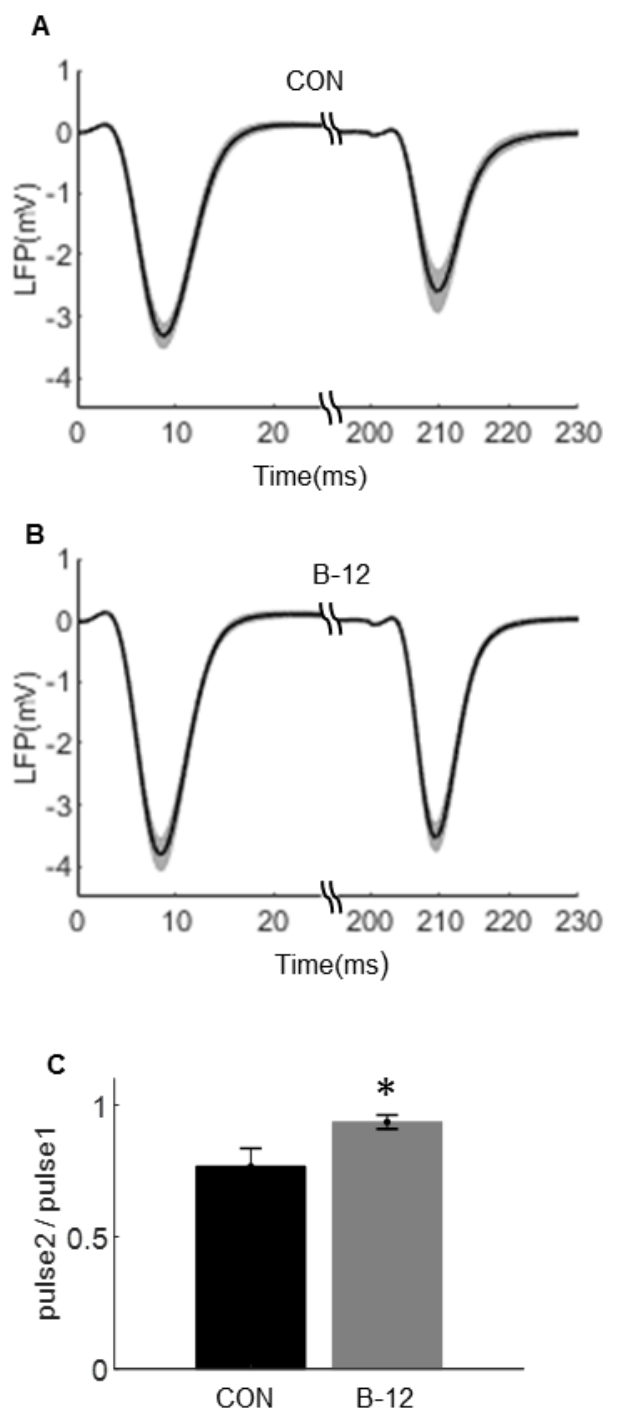
Figure 4

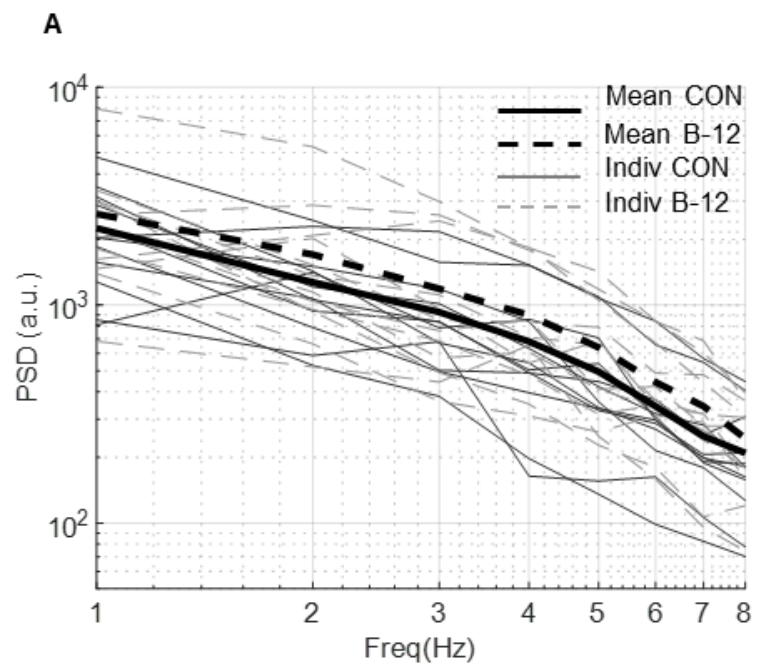

B

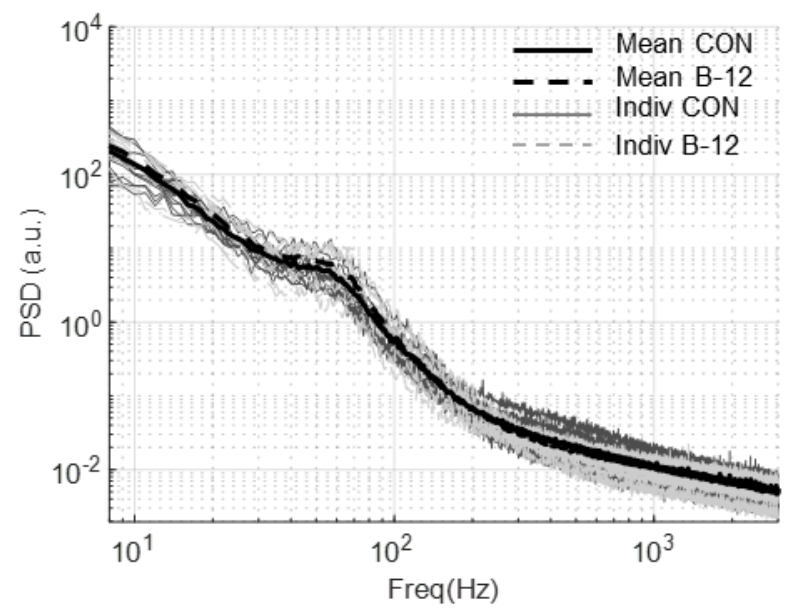


Figure 5
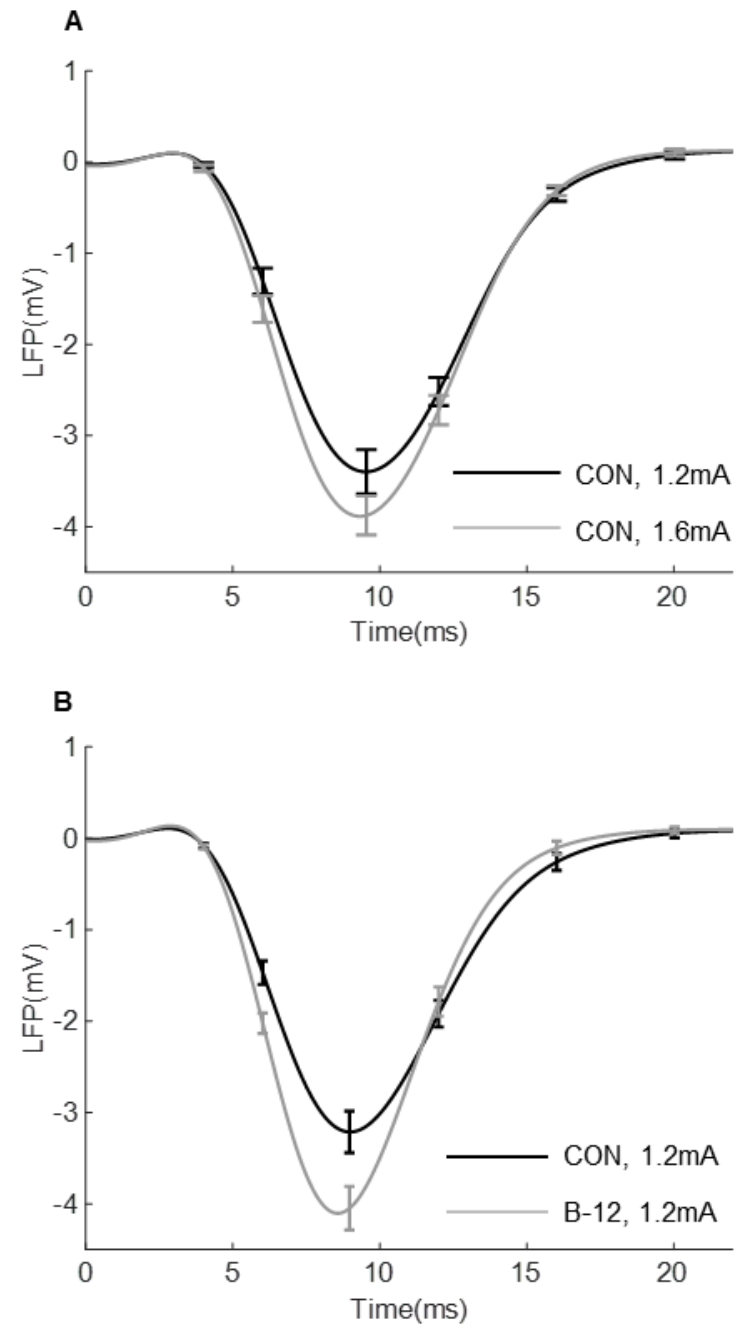